\title{
Resignificando la masculinidad hegemónica: reflexiones y prácticas de hombres universitarios ${ }^{1}$ \\ Resignifying hegemonic masculinity: reflections and practices of university men
}

pp. $60-75$

LUISA JOHANNA ARIAS TAPASCO ${ }^{2}$

Carolina Centeno Perea ${ }^{3}$
REC: $16 / 03 / 2021$

ACEP: $19 / 05 / 2021$

\section{Resumen}

El presente artículo se deriva de la investigación desarrollada en el 2020, que tuvo como propósito la comprensión de los factores socioculturales que inciden en la crítica y resignificación de la masculinidad en estudiantes de Derecho, Comunicación Social y Trabajo Social de Unicatólica. Se retomaron algunos postulados de la fenomenología y la construcción social de la realidad como paradigma y teoría que permitieron comprender la realidad de los estudiantes y los significados que le otorgan a su masculinidad. Metodológicamente fue un estudio descriptivo con enfoque cualitativo, que precisó un muestreo por conveniencia; se implementaron entrevistas semiestructuradas, un grupo de discusión, técnica interactiva (fotopalabra) y cuestionario virtual como técnicas para la recolección de datos. En los resultados se destaca la influencia del contexto sociocultural y universitario en la construcción de la masculinidad; así mismo, se ilustra la necesidad de continuar con estudios que analicen las diferentes formas en las que se configura la masculinidad desde una perspectiva relacional.

1. Artículo producto de investigación en el marco del Semillero Semillas de Innovación. Agradecemos a los jóvenes participantes del estudio, al programa académico de Trabajo Social y a la Unidad de Investigaciones de la Fundación Universitaria Católica Lumen Gentium, por su apoyo para la realización de este proyecto.

2. Trabajadora Social, Universidad del Valle; Magíster en Salud Pública, Universidad del Valle; docente, Fundación Universitaria Católica Lumen Gentium - Unicatólica, Santiago de Cali, Valle del Cauca, Colombia. Correo electrónico: larias@unicatolica.edu.co - Orcid: https:// orcid.org/0000-0001-9221-6068

3. Trabajadora Social, Universidad del Valle; Magíster en Intervención Social, Universidad del Valle; docente, Fundación Universitaria Católica Lumen Gentium - Unicatólica, Santiago de Cali, Valle del Cauca, Colombia. Correo electrónico: ccenteno@unicatolica.edu.co Orcid: https:// orcid.org/0000-0003-4040-1506 
Palabras clave: masculinidad, género, factores socioculturales, resignificación, educación.

\section{Abstract}

This article is derived from the research developed in 2020 whose purpose was to understand the socio-cultural factors that affect the criticism and resignification of masculinity in students of Law, Social Communication and Social Work at Unicatólica. Some postulates of phenomenology and the social construction of reality were retaken as a paradigm and theory that allowed us to understand the reality of the students and the meanings that they give to their masculinity. Methodologically it was a descriptive study with a qualitative approach, it required a convenience sampling; semi-structured interviews, a discussion group, interactive technique (photoword) and virtual questionnaire were implemented as techniques for data collection. The results highlight the influence of the sociocultural and university context in the construction of masculinity, likewise, the need to continue with studies that analyze the different ways in which masculinity is configured from a relational perspective is illustrated.

Keywords: masculinity, gender, sociocultural factors, resignification, education.

\section{Introducción}

En el presente artículo se describe el proceso y los resultados alcanzados en la investigación "Factores socioculturales que influyen en la resignificación de la masculinidad en estudiantes de la Facultad de Derecho, Ciencias Sociales y Políticas (FDCSP) de UnicatólicaCali", que estuvo orientada a la generación de nuevos espacios de diálogo y conocimiento en el ámbito universitario, lo que a mediano y largo plazo aportará a las transformaciones en las dinámicas académicas y culturales de los jóvenes desde las cuales se piensen lo que significa ser hombre y mujer en esta sociedad.

La investigación se desarrolló desde un enfoque cualitativo, con el fin de comprender la resignificación de masculinidad en estudiantes de la FDCSP de Unicatólica, siendo esto clave para la generación de procesos pedagógicos y de intervención que giren en torno a las formas hegemónicas de asumir la masculinidad y las violencias que de allí se configuran en las relaciones sociales cotidianas, teniendo en cuenta que las construcciones de ser hombre o mujer están relacionadas con los contextos socioculturales e históricos en los cuales se desarrollan.

Así mismo, se presentan los principales antecedentes que dieron sustento al planteamiento del problema, la pregunta de investigación y los objetivos tanto general como específicos; a partir de lo anterior, se desarrollan las claves teórico-conceptuales que orientaron el estudio, la estrategia metodológica que permitió la gestión y análisis de la información; se finaliza con algunas aproximaciones al análisis y discusión de los principales hallazgos, las conclusiones y recomendaciones derivadas del estudio.

\section{Antecedentes}

Con el fin de reconocer los principales debates, teorías y metodologías para la comprensión y abordaje de las masculinidades, se realizó una búsqueda en bases de datos, tales como Web of Science del Instituto Internacional de Estadística, Google Scholar, Scopus y Sciencedirect; tomando como referencia las siguientes palabras clave: 
masculinidad hegemónica, masculinidades, perspectiva de género, sociocultural. La temporalidad de la producción académica revisada se ubicó entre los años 2000 al 2019.

Dentro de la revisión realizada en el marco de las ciencias sociales, se aprecia que uno de los referentes importantes desde donde se ha discutido e investigado la masculinidad, su construcción, su crítica y su perspectiva, además de su especificidad, es justamente el de los estudios de género, que incluye miradas disciplinares específicas. En este sentido, se tiene que, ya sea desde un campo específico como el de los estudios de género y masculinidad (Faur, 2004; Ortega et al., 2005; Instituto Vasco de la Mujer [Emakunde], 2008; Aguayo et al., 2011; Heilman et al., 2017) 0 desde miradas disciplinares específicas como la sociología (Peña, 2008; Villanueva, 2011; Sanfélix, 2011; Saavedra, 2013; Sandoval, 2014; Muñoz, 2015), la psicología (de Sola et al., 2003; Ale, 2010; Barba y Gómez, 2016; Medina et al., 2018) y también la antropología y el trabajo social (Téllez y Verdú, 2011; Castro, 2016; Muñoz, 2015), se recogen puntos de vista que se articulan con el problema de investigación, el cual parte desde la mirada del sentido y significado que las prácticas cotidianas tienen en la construcción y reconstrucción de las identidades de género en hombres. Así mismo, las investigaciones revisadas dan cuenta de una mirada sobre el fenómeno desde una construcción social siendo acorde con la propuesta presentada, de manera que ella permite entender que las identidades en torno al género son producto de contextos sociales y de la interacción entre ellos y los sujetos.

En esta línea se ubica lo propuesto por Messerschmidt (2010), quien refiere una tendencia intelectual de "leer" la masculinidad hegemónica desde un carácter estático, ignorando de esta manera toda la cuestión de dinámica de los géneros (Connell, 2008, como se citó en Messerschmidt, 2010). El autor argumenta que es invaluable para los estudios de género aumentar y diversificar la investigación sobre cómo las relaciones patriarcales se legitiman y estabilizan a nivel local, regional y global (especialmente los dos últimos niveles) y cómo cada nivel impacta a los otros niveles, además cómo estas masculinidades hegemónicas están relacionadas con las masculinidades no hegemónicas. De igual manera, se requiere de trabajos académicos que permitan avanzar en la comprensión sobre cómo varios grupos subordinados (no solo mujeres) ayudan (o no) a cultivar la masculinidad hegemónica y cómo tal desigualdad puede ser cuestionada y disputada legítimamente.

En el estudio de Manzelli (2006) se buscó explorar y analizar los significados que tienen los jóvenes adolescentes sobre ser hombre y la influencia de estos en sus interacciones. Dentro de los hallazgos, el autor alude a que los significados analizados se interrelacionan con pautas culturales que permiten interpretar algunos comportamientos asimétricos en las relaciones de género; es importante aclarar que se encontraron algunas diferencias en relación con el estrato socioeconómico, la edad y el nivel educativo de los entrevistados. Ahora bien, en lo que tiene que ver con los significados sobre qué es ser hombre y qué es ser mujer, se encontraron algunos adolescentes que identificaron las inequidades de género, lo que lleva a pensar que hay alternativas desde las cuales se pueden construir las identidades masculinas diferentes a las identidades que tradicionalmente se han visto desde las posturas y relaciones de desigualdad.

En el artículo de Flecha et al. (2013) se presenta un análisis de los efectos en la violencia 
contra las mujeres desde las Masculinidades Tradicionales Dominantes (DTM), Masculinidades Tradicionales Oprimidas (OTM) y las Nuevas Masculinidades Alternativas (NAM). La principal conclusión de los autores es que las OTM son complementarias a las DTM, dado que son dos caras de la misma moneda. Al contrario, las NAM efectúan una contribución clave para la superación de la violencia contra las mujeres. Las evidencias proporcionadas por las investigaciones también indican que las actuaciones llevadas a cabo en programas basados en el citado descubrimiento, de la misma manera están contribuyendo a la superación de la violencia contra las mujeres.

De otro lado, Gittings (2016), a partir de entrevistas semiestructuradas y en profundidad, exploró las técnicas que los trabajadores de atención comunitaria en VIH emplean cuando trabajan con pacientes masculinos diagnosticados con VIH; en el desarrollo metodológico de la investigación se realizaron visitas de observación al hogar, que permitieron considerar la dinámica entre los pacientes y los trabajadores de atención comunitaria. Así mismo, se realizaron entrevistas con dos investigadores y activistas del VIH para triangular los resultados de la investigación. Como conclusiones Gittings (2016) señaló que tanto los pacientes como los trabajadores se topan con los ideales y normas de lo que significa ser un hombre, lo que deriva en que sea más difícil para los pacientes hombres acceder a la atención necesaria porque es visto como un signo de debilidad y feminidad, mientras que trabajadores de atención se esfuerzan por brindar atención y recibir apoyo, además de vencer las prácticas que desde la masculinidad inhiben la confesión y el apoyo.

En el contexto latinoamericano la autora Mara Viveros (2002), con trayectoria amplia en el tema, agrupó en tres ejes los diferentes estudios sobre masculinidades, lo que da cuenta de la pluralidad de tendencias y comprensiones a propósito del tema:

- Identidades masculinas: los significados de la masculinidad, los efectos del contexto social en las identidades masculinas, identidades masculinas en el mundo del trabajo, masculinidades y clases sociales, masculinidades e identidades étnico-raciales;

- Las masculinidades en el ámbito privado: la paternidad, prácticas y representaciones, los estudios sobre salud sexual y reproductiva, las fronteras de la sexualidad;

- Violencias y homosocialidad masculina: masculinidades y violencia, escenarios de homosocialidad masculina.

En Colombia se identificó el estudio realizado por Catacolí et al. (2018), quienes intentaron dar respuesta a las configuraciones de las relaciones de género en Unicatólica, a partir de una línea de base que permitió identificar las particularidades de la comunidad educativa respecto a dichas relaciones en clave de conocimientos, actitudes y prácticas que inciden en el fenómeno abordado, aportando de esta manera elementos para la consolidación de campañas que incentiven reflexiones acerca de las consecuencias de la cultura patriarcal y las desigualdades de género.

De otro lado, Tovar y Pavajeau (2010) dan cuenta de la falta de reflexión académica y política acerca de la situación de los hombres en condición de desplazamiento forzado en Colombia, de ahí que analizaron cómo esta problemática incide en la reconfiguración de las masculinidades, al tiempo que produce tensiones, reacciones y transformaciones en su subjetividad de género, además de mencionar las implicaciones sociales de dicha 
situación, invitando a pensar el tema desde una perspectiva psicosocial para el diseño de estrategias de acompañamiento útiles para avanzar hacia la equidad de género en este campo.

En la investigación de Burbano et al. (2019) se aborda la construcción de masculinidades en las narrativas de once motociclistas en Valledupar-Colombia; a partir de metodología cualitativa, los autores concluyen que las masculinidades de los entrevistados están "en tránsito"; es decir, oscilando entre las manifestaciones hegemónicas y la aparición de otras formas de ser y convertirse en un hombre. Con base en el análisis de las historias biográficas de los participantes, se evidenció que en su territorio su masculinidad se cuestiona constantemente, se prueba repetidamente en el entorno social, una razón para la necesidad de una demostración permanente.

A partir de lo reseñado líneas atrás, se evidencia una clara orientación metodológica cualitativa con algunas excepciones en las cuales el método apunta a una elaboración estadística (Aguayo et al., 2011; Heilman et al., 2017; Sagaró y Moraga, 2015; de Sola et al., 2003). Lo cualitativo emerge como la manera más adecuada para afrontar estos temas, pues considera las vivencias y relatos de las personas antes que generalizar 0 establecer patrones comunes, aunque esto no significa que el uso de encuestas no sea viable para complementar el análisis bajo este paradigma.

En cuanto a las herramientas para el desarrollo de las investigaciones reseñadas, se aprecia que los relatos e historias de vida, el análisis documental y la etnografía, son metodologías importantes para dar cuenta de la construcción y cambios en las masculinidades, a la vez que técnicas como las entrevistas (semi-estructuradas y en profundidad), los grupos focales y de discusión, resultan útiles para darles sentido a los contextos y prácticas sobre los que se afirman las identidades culturales y de género.

De igual manera, se alude al género como categoría de análisis relacional que permite el abordaje analítico y crítico sobre las relaciones entre mujeres y hombres, señalando la relevancia de estas relaciones en la estructuración de las sociedades humanas.

Respecto al contexto de las investigaciones, se encontró que hay experiencias tanto a nivel nacional (Tovary Pavajeau, 2010; Catacolí et al., 2018; Barba y Gómez, 2016; Castro, 2016; Faur, 2004; Medina et al., 2018; Muñoz, 2015) como internacional (Manzelli, 2006; Messerschmidt, 2010; Wilson et al., 2013; Gittings, 2016; Burbano et al., 2019; Gökariksel y Secor, 2017; Aguayo et al., 2011; Ale, 2010; Saavedra, 2013; Villanueva, 2011) y el referido a España (Instituto Vasco de la Mujer [Emakunde], 2008; Peña, 2008; Sanfélix, 2011; de Sola et al., 2003), donde se priorizó el contexto latinoamericano (Aguayo et al., 2011; Ale, 2010; Saavedra, 2013; Villanueva, 2011), con el fin de reconocer las condiciones socioculturales que inciden en las construcciones y significaciones a propósito de las masculinidades, lo que evidencia la necesidad de explorar desde la interseccionalidad (clase, raza y lo étnico) y la globalización, las dinámicas, fisuras y continuidades a propósito de las masculinidades hegemónicas y no hegemónicas.

De igual manera, se puede evidenciar que las sociedades contemporáneas han logrado establecer el tema de la igualdad de género como un aspecto central en la agenda de los gobiernos y como una preocupación constante desde la opinión pública. En este sentido, 
muchas de las acciones que se han adelantado en esta dirección tienden a ampliar los espacios de participación y acción que tienen las mujeres; sin embargo, esto también ha conllevado a que, en vez de garantizar espacios de convivencia y aceptación desde la igualdad de género, se terminan evidenciando actitudes e imaginarios por parte de los hombres desde sus roles tradicionales e ideologías dominantes. Esto es evidente en nuestra sociedad colombiana, donde diariamente se dan a conocer casos de violencia de género, tanto en la cotidianidad de las personas como en los medios de comunicación, los cuales son claramente signos de una sociedad donde la idea de hombre está fuertemente vinculada al ejercicio de la violencia contra la mujer (física, económica, simbólica, etc.) y donde es urgente generar espacios y propuestas que lleven a la reflexión sobre estas prácticas y los contextos que las promueven.

Es aquí donde se hace necesario repensar la igualdad de género por medio de la crítica a los modelos hegemónicos de construcción de identidad en los hombres, de manera que aquello que define a la masculinidad desde la historia y la cultura pueda ser discutido y resignificado desde los espacios académicos y políticos donde se proponen los modelos de sociedad.

\section{Consideraciones teórico-conceptuales}

Para la construcción del marco de referencia teórico en el que se apoyó la investigación fue necesario realizar un rastreo de las claves teóricas desde las cuales se han comprendido las masculinidades no hegemónicas, dado el carácter social, variable y construido de estas. En este sentido, tal como lo señala Viveros (2002, p. 53, citando a de Barbieri, 1992; Gomariz, 1992 y Hearn, 1987, entre otros), es necesario ubicar los estudios sobre masculinidades en el campo de los estudios de género como resultado del avance de la teoría feminista, de la constatación por parte de numerosos autores de la invisibilidad en las ciencias sociales del varón como actor dotado de género y de los cuestionamientos de los privilegios masculinos, provenientes de los movimientos feministas.

Además de lo anterior, se tomó como referencia el paradigma fenomenológico y la teoría de la construcción social de la realidad, permitiendo la comprensión del objeto de estudio. De manera general, la fenomenología se interesa por los procesos que constituyen intersubjetivamente las estructuras de la realidad y la forma como se construye y reconstruye permanentemente la vida social (Schutz y Luckmann, 2003).

Igualmente, es pertinente mencionar, de acuerdo con Berger y Luckmann (1986), que la realidad social es una construcción social, en donde el lenguaje objetiviza el pensamiento del sujeto, a la vez que trasciende las barreras temporales y espaciales, posibilitando materializar actos y situaciones de la vida cotidiana.

Por tanto, los seres humanos se constituyen como seres sociales que se encuentran en constante interrelación con el contexto que los rodea y tienen la capacidad de ser creadores y protagonistas de sus vidas; se construyen día a día en la búsqueda continua por realizarse en diversas áreas de sus vidas (trabajo, familia, vecindario, amigos, etc.). De esta manera, cuando los sujetos interactúan, construyen a partir de sus visiones y acciones, la realidad que les rodea; de allí que esta se conciba como una construcción social, que puede ser sostenida o transformada. 
Teniendo en cuenta las anteriores claridades teóricas, se conceptualizan como categorías centrales en el estudio la construcción de significados y los factores socioculturales. Al respecto, Bruner (1991) planteó que los sujetos les otorgan significado a sus experiencias y a partir de allí se construye la realidad, por ello la realidad no está por fuera de los sujetos, sino que es una construcción en la cual influyen las construcciones mentales y las creencias del individuo; no obstante, la construcción de significado va a depender también de la relación con los otros y de factores socioculturales, los cuales se comprenden como aquellos aspectos que dan cuenta de las formas de organización, los estilos de vida, las ideologías, los imaginarios, las normas, las costumbres, las expectativas y los roles según el género, en un momento histórico determinado.

En este sentido, se vincula al género como categoría de análisis relacional, que permite entender cómo dichos factores operan en cada contexto, cuáles son los códigos éticos y políticos que lo sostienen, cómo se consolida lo correcto, la norma, el deber ser en las subjetividades y colectividades hasta naturalizarlo, anclando así una visión crítica que facilita pensar en la necesidad de una deconstrucción cultural de los géneros (Pavajeau, 2006, como se citó en Tovar y Pavajeau, 2010, p. 96).

En este orden de ideas, se aprecia la conexión entre los factores socioculturales y la construcción de significados, dado que estos posibilitan la emergencia de prácticas relacionadas con lo socialmente establecido respecto a ser hombre y ser mujer; dichas prácticas se comprenden como productos de aprendizajes. Se retoma lo propuesto por Bourdieu (1991) en relación con la idea de práctica, ya que desde sus postulados se expone inicialmente el habitus como disposiciones duraderas y transferibles, como principios generadores y organizadores de prácticas y representaciones que pueden estar objetivamente adaptadas a su fin, sin la búsqueda consciente de fines y el dominio expreso de las operaciones necesarias para alcanzarlos; sin embargo, no se excluye que las respuestas del habitus vayan acompañadas de un cálculo estratégico. De este modo, el mundo práctico que se constituye en la relación con el habitus, es un mundo de fines ya realizados de caminos por seguir.

Finalmente, en las consideraciones teórico-conceptuales, se retomó la categoría de masculinidad hegemónica que ha influenciado el pensamiento reciente sobre los hombres, el género y la jerarquía social (Connell y Messerschmidt, 2005). Dicha categoría proporcionó un vínculo entre el creciente campo de investigación de los estudios de hombres (también conocidos como estudios de masculinidad y estudios críticos de hombres), los relatos feministas del patriarcado y los modelos sociológicos de género.

La masculinidad hegemónica como concepto alude al conjunto de atributos, valores, funciones y conductas que se suponen esenciales al hombre en una cultura determinada, aunque no haya una sola manera de definirla y se le atribuyen diferentes características a la persona que se considera masculina dependiendo del enfoque que se tenga. (Sandoval, 2014, p. 58)

Sumado a ello, se reconoce el carácter múltiple de la masculinidad, lo que pone en evidencia la necesidad de comprender la relación entre las mismas y las diferencias que subyacen a partir de variables como raza, etnia y clase. De igual manera, en la comprensión de las masculinidades, se tiene en cuenta la importancia no solo de los comportamientos sino también de los discursos, mostrando cómo a través de ellos se presenta, defiende 
y justifica la posición hegemónica de los varones (Viveros, 2002).

A partir de los aspectos referenciados antes, se propuso dar respuesta al siguiente interrogante: ¿Cuáles son los factores socioculturales que permiten a estudiantes de la Facultad de Derecho, Ciencias Sociales y Políticas (FDCSP) de Unicatólica, la crítica y resignificación de su masculinidad? En coherencia con lo anterior, se planteó como objetivo general: comprender los factores socioculturales que permiten la crítica y resignificación de la masculinidad en estudiantes de la Facultad de Derecho, Ciencias Sociales y Políticas de Unicatólica. Para dar respuesta a dicho objetivo, se trazaron los siguientes objetivos específicos: 1) identificar las expectativas y conocimientos a partir de los cuales han construido su masculinidad los estudiantes de la FDCSP de Unicatólica; 2) explorar las relaciones de intercambio que posibilitan la crítica de la masculinidad hegemónica en los estudiantes de Unicatólica; y 3) caracterizar las prácticas de los estudiantes que dan cuenta de la apropiación de masculinidades no hegemónicas.

\section{M etodología}

Se realizó una investigación de tipo descriptivo y se retomó el enfoque cualitativo a partir de un diseño fenomenológico, con el fin de explorar, describir y comprender los significados y experiencias de los estudiantes de la FDCSP sobre la masculinidad y las formas en que se acercan o se distancian de las expectativas, discursos y prácticas respecto a ser hombre desde una mirada hegemónica, descubriendo de esta manera los elementos en común (Hernández, 2014).

Con el fin de definir la cantidad de sujetos que se vincularon al estudio, se hizo una caracterización preliminar de los estudiantes de Derecho, Trabajo Social y Comunicación Social-Periodismo matriculados en el año 2020 , esto con el fin de conocer datos sociodemográficos tales como edad, estado civil, pertenencia étnica, estrato socioeconómico; no obstante, el muestreo utilizado fue por conveniencia, es decir, aquel conformado por los casos disponibles y a los cuales se tiene acceso; este tipo de muestreo es no probabilístico, lo que indica que más allá de una representatividad o generalización en términos estadísticos, lo que se busca es información guiada por las características y razones propias de la investigación (Hernández, 2014).

Todos los participantes del estudio fueron mayores de 18 años, quienes aceptaron la participación voluntaria, previa lectura del consentimiento informado en donde se precisó información sobre el alcance de este, la confidencialidad y el uso de la información. El trabajo de campo se inició en el mes de julio del 2020, a partir de la implementación de técnicas de recolección de datos, tales como: grupo de discusión, cuestionario virtual, entrevista semiestructurada y la técnica interactiva fotopalabra. El proceso de análisis de la información se desarrolló a partir de un ejercicio de categorización inductiva, previa validación y ajustes de los instrumentos de recolección; posteriormente, se realizó la transcripción de la información y teniendo en cuenta las categorías de análisis, se procedió con la descripción, análisis e interpretación de los datos, considerando el andamiaje teórico y conceptual del estudio.

El número de estudiantes que se vincularon a cada una de las técnicas que se implementaron se aprecia en la Tabla 1. En relación con el porcentaje de participación de los estudiantes teniendo en cuenta la pertenencia a los programas académicos de la FDCSP, se 
Tabla 1

Asistencia estudiantes por técnica.

\begin{tabular}{|c|c|}
\hline Técnica de recolección de datos & No. asistentes \\
\hline Cuestionario virtual & 22 \\
\hline Grupo de discusión & 9 \\
\hline Entrevista semiestructurada & 13 \\
\hline Técnica interactiva & 9 \\
\hline
\end{tabular}

Fuente: Elaboración propia.

evidenció que predominaron los participantes del programa Trabajo Social, como se muestra en la Figura 1.

\section{Figura 1}

Participación de estudiantes por programa académico.



Fuente: Elaboración propia.

Respecto a la información sociodemográfica que se logró obtener a través del cuestionario virtual, el $77,3 \%$ de los estudiantes se ubica entre 16 y 26 años, el $95,5 \%$ son solteros y no tienen hijos, en cuanto a la pertenencia étnica el $31,8 \%$ se reconocen como negros y $68,2 \%$ como mestizos; todos viven en la ciudad de Cali. En relación con la ocupación laboral, el $50 \%$ refirió estar laborando; de dicho porcentaje el $63,6 \%$ trabajan como empleados y el resto como independientes $(36,4 \%)$.
Sobre los factores socioculturales que influyen en la resignificación de la masculinidad: apuntes analíticos y discusión

A continuación se presentan los hallazgos principales de la investigación que dan cuenta de la comprensión de los factores socioculturales que posibilitan la crítica y resignificación de la masculinidad por parte de los estudiantes y algunas investigaciones que sirven de base para la discusión.

Uno de los principales hallazgos del estudio demuestra que los estudiantes asumen la diferenciación biológica como característica asociada a lo masculino, además del predominio de la fuerza física que deberían tener los hombres, como se aprecia en la siguiente verbalización:

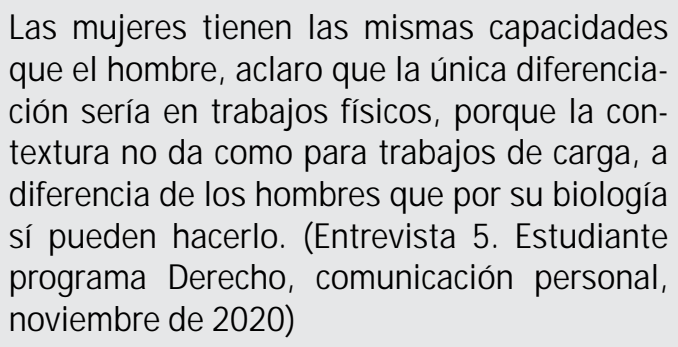

Las mujeres tienen las mismas capacidades que el hombre, aclaro que la única diferenciación sería en trabajos físicos, porque la contextura no da como para trabajos de carga, a diferencia de los hombres que por su biología sí pueden hacerlo. (Entrevista 5. Estudiante programa Derecho, comunicación personal, noviembre de 2020)

Sumado a lo anterior, se reconoce la relevancia del andamiaje simbólico-cultural en los significados asociados a la masculinidad:

...nos hace masculinos, además de toda la carga genética que podemos traer, todas esas construcciones que hemos hecho a lo largo de toda una vida, entonces hay acciones, comportamientos, actitudes, formas de pensar, aspectos de personalidad que podrían orientar, todas juntas, a definir que un ser humano es un ser masculino. (Entrevista 6. Estudiante programa Trabajo Social, comunicación personal, diciembre de 2020) 
De igual manera, en la técnica interactiva desarrollada, al mostrar las imágenes los participantes aludieron a la temática de la masculinidad expresando que:

Al mirar la imagen, se propone un cuestionamiento, hay una masculinidad única; quienes se salen de dicha masculinidad, son anormales. Eso es lo que quiere sugerir la imagen, se debe cuestionar esa idea tóxica de masculinidad, es importante revisar las ideas que desde la iglesia y la sociedad se han impuesto. (Técnica interactiva Fotopalabra, Estudiante programa Trabajo Social, comunicación personal, noviembre de 2020)

Este hallazgo ya ha sido reportado en la literatura; por ejemplo, un estudio de Manzelli (2006) en Buenos Aires, Argentina, exploró y analizó los factores sociales y culturales que intervienen en los significados que construyen jóvenes sobre el ser hombre y las implicaciones que tienen estos significados en sus interacciones sociales. En dicho estudio se muestran resultados similares en tanto la fuerza física se asume como uno de los aspectos diferenciales entre hombres y mujeres. Igualmente, esta se asocia con la rudeza y la preparación para el mundo del trabajo por el lado masculino, siendo una ventaja para los hombres.

Otro de los resultados permite evidenciar que la genitalidad no determina el desempeño de actividades y roles; en sus discursos los participantes indican que el hombre puede desarrollarse de forma libre como cualquier persona en sociedad, sin necesidad de encasillarse en los roles impuestos socialmente; ejemplo de ello es el reconocimiento de que tanto hombres como mujeres pueden desarrollar las mismas ocupaciones, prácticas deportivas y profesiones, dado que cuentan con las capacidades y habilidades para ello; entonces, estas no están predeterminadas por el género, pese a lo socializado tradicionalmente. En algunos de sus discursos se expone lo siguiente:

no hay funciones específicas ni para el hombre ni para la mujer, el hombre puede lavar en la casa, el hombre puede trapear en la casa, el hombre puede atender sus hijos; ehhh, la mujer puede hacer lo mismo que el hombre, para mi no hay una función específica que el hombre y la mujer deben realizar en la casa.. pienso que como personas nos han codificado a cumplir con unos roles, que realmente para mí han traído muchos problemas. (Entrevista 1. Estudiante programa Trabajo Social, comunicación personal, julio de 2020)

En relación con las nociones de sexualidad y género, a partir de los resultados se evidenció que la mayoría de los estudiantes asocian la sexualidad con las características biológicas y psicosociales del ser hombre y ser mujer; respecto al género, los estudiantes en su mayoría lo reconocen como una categoría que da cuenta de las construcciones sociales sobre ser hombre y ser mujer; no obstante, llama la atención que dicho concepto también fue asociado a la diferenciación biológica de hombres y mujeres. De acuerdo con lo anterior, se aprecian ciertas limitaciones respecto a la comprensión de la sexualidad, pues esta trasciende la diferenciación biológica; es decir, es un aspecto fundamental de la condición humana, presente a lo largo de la vida, abarcando no solo la genitalidad sino las identidades y los roles de género, la orientación sexual, el erotismo, el placer y la intimidad (OPS, 2018).

Otro de los hallazgos alude al contraste entre los discursos y las prácticas de los estudiantes en relación con los roles y la posición social de privilegio que históricamente han tenido los hombres en la sociedad; no obstante, este cuestionamiento no trasciende a 
acciones concretas que permitan evidenciar la igualdad entre hombres y mujeres. Lo anterior también fue evidenciado en el análisis de Muñoz (2015), quien realizó un estudio en Medellín (Colombia) con el fin de abordar la construcción y significación de las masculinidades en hombres heterosexuales. Este autor reconoció, a partir de los relatos de los participantes, discursos que muestran la importancia de algunos cambios en las relaciones de género, especialmente en la participación de las mujeres en el espacio público, la admisión de temas como la no violencia contra las mujeres; sin embargo, estos hombres aún no logran materializar conductas que verdaderamente posibiliten la igualdad, pues aún se reservan algunos privilegios de orden desigual entre hombres y mujeres.

Si bien los estudiantes que participaron en el estudio cuestionan los principales roles y discursos que se les han impuesto a través de la socialización, dicho cuestionamiento no es sinónimo de la transformación total en sus acciones; es decir, identifican la necesidad de cambiar algunas acciones en función de trascender los modos tradicionales y condicionados de "ser varón". Sin embargo, en su cotidianidad siguen replicando algunas acciones de las que quisieran distanciarse; por ejemplo, cohibirse en la expresión de sentimientos, pese a lo anterior buscan cambiar y trascender a prácticas en las cuales logren expresar sentimientos de manera asertiva; lo anterior fue reflejado por uno de los participantes al preguntarle sobre la expresión de sentimientos en su familia:

Yo diría que ahí sentimientos prácticamente no hay, y yo pienso romper con ese ciclo, de no ser afectivo, porque creo que eso a usted no lo hace ni menos, ni más hombre, solamente usted expresa ante su familia lo que quiere; de hecho, yo he derrumbado una barrera con mi padre, porque él no es de abrazar, en cambio yo sí, como que venga un abrazo, es que usted es mi papá, no solamente es dinero, no solamente es hablar, no sé, digamos que aunque yo no conviva con él, no solamente es como que eso, he tratado de romper esas barreras; sin embargo, están presentes. (Entrevista 4. Estudiante programa Comunicación Social, comunicación personal, noviembre de 2020)

Igualmente se apreció un discurso relacionado con la presión social que pueden experimentar los hombres al momento de expresarse, tal como lo refirió un entrevistado:

creo que uno se siente vulnerable frente a
otras personas por mostrar las debilidades,
pero estoy consciente de que son pensamien-
tos que uno se deja influenciar por la socie-
dad, porque uno no está cohibido por expre-
sar lo que está sintiendo, pero como te decía
uno se deja permear y ahí es donde vienen los
pensamientos de "me siento vulnerable ante
el otro por el simple hecho de mostrar mis sen-
timientos". (Entrevista 3. Estudiante programa
Comunicación Social, comunicación personal,
agosto de 2020)

Este resultado es comparable con el estudio realizado en Estados Unidos, México y Reino Unido, en el cual Heilman et al. (2017) buscaron estudiar lo que significa ser un hombre joven, cómo se supone que deben actuar y qué tanto peso tienen las expectativas sobre el ser hombre en sus vidas. Como resultado estos autores señalan que en el plano emocional los hombres sienten temor a ser vulnerables, por lo que la mayoría de las veces afrontan de manera individual sus emociones 0 acuden al apoyo de las mujeres presentes en sus vidas.

De otro lado, al abordar las relaciones de intercambio que han permitido la crítica de la masculinidad hegemónica por parte de los estudiantes, se aprecia la influencia que ejerce la familia en la construcción de su identidad 
como hombres, además de la importancia que se les otorga a los hábitos familiares y a las enseñanzas de las labores del hogar por parte de personas con las que han convivido (padres, madres, hermanos/as, etc.). Un estudiante del programa de Derecho mencionó al respecto:

En el hogar no hay ningún oficio que desacredite como tal la hombría de uno como persona, puede cocinar, puede lavar los trastes, puede hacer el aseo, lavar los baños, eso no quita lo hombre. La verdad entre mi familia siempre se manejó eso (labores del hogar). (Entrevista 2. Estudiante programa Derecho, comunicación personal, agosto de 2020)

Respecto a las relaciones de pareja, los estudiantes señalan la importancia de no dejar de lado aspectos relacionados con manifestaciones afectivas de diversa índole; aunque dichas prácticas están mediadas por el contexto sociocultural.

Ahora bien, en cuanto a las relaciones con los pares, en su mayoría los estudiantes indican que dialogan con sus amigos hombres de temas como el entorno laboral, la situación económica y relaciones afectivas; por otra parte, en estos diálogos no existe una tendencia a mencionar asuntos como las frustraciones sexuales, temas familiares, ni aspectos de moda.

De igual manera, algunos de los estudiantes entrevistados afirman que los discursos y temas que sostienen con sus amigos hombres son diferentes en comparación con los de sus amigas mujeres; aunque algunos hacen la aclaración que dicha diferencia también depende del vínculo, es decir, que independientemente de sostener conversaciones diferentes con hombres o mujeres, se le da relevancia al tipo de relación y confianza que tienen con sus amigos y amigas.
Sobre la satisfacción con la vida y el rol que asumen como hombres, los estudiantes reconocen la necesidad de seguir avanzando en la transformación de la ideología patriarcal:

creo que los hombres hemos estado en una posición social, política e histórica de privilegio que nos debemos cuestionar, toda vez que ha violentado a las mujeres. (Cuestionario virtual, estudiante Trabajo Social, comunicación personal, octubre de 2020)

Esto con el fin aportar a la equidad e igualdad entre los géneros, logrando un equilibrio frente a lo que desean y los ideales que se han construido en la sociedad respecto a la masculinidad. Con todo lo anterior, es pertinente retomar el planteamiento de Barba y Gómez (2016):

la masculinidad permanentemente se está transformando ya por accidentes históricos, ya por propósitos conscientes-colectivos, el momento actual permite observar una transformación evidente hacia construcciones de género más orientadas a la equidad, reconociendo, sin embargo, que aún hay mucho por avanzar (...) (p. 257)

En este sentido, se comprende que los imaginarios y expectativas sobre la masculinidad hegemónica no han desaparecido del contexto; es decir, confluyen con otras masculinidades. Sumado a esto, un estudiante refirió:

Algo que he logrado apreciar en mi pueblo (...) yo no podía expresarme de cierta manera, siempre viví reprimiendo mis sentimientos. La frase va muy relacionada con el tipo de creencia que se maneje en la familia. La sociedad los ve como unos fracasos, varias personas me han dicho sentimental, la hegemonía del hombre, es el fuerte, el valiente, especialmente en contextos vulnerables, donde el hombre tiene que mostrar como ese enojo, porque si 
demuestra lo contrario, lo van a ver como débil; lastimosamente lo he vivido y he visto que pasa así. (Grupo de Discusión, , comunicación personal, noviembre de 2020)

A su vez, se destaca el aporte de la academia en la resignificación de la masculinidad, pues los estudiantes señalan diferentes ideas que incentivan la reflexión y el cuestionamiento de esta, como se evidenció en lo expresado por uno de los participantes:

Sé que socialmente se nos "imponen" una serie de prácticas como hombres, creo que también la academia hace que podamos ampliar nuestro panorama y pensarnos si realmente estas prácticas son correctas o no y creo que de una u otra manera he ido creando mi propio rol de hombría. (Cuestionario virtual, estudiante Trabajo Social, , comunicación personal, octubre de 2020)

En este orden de ideas, es importante resaltar el valioso aporte del contexto universitario, pues permite que los estudiantes tengan diferentes comprensiones sobre lo que significa ser hombre y participen de los escenarios de diálogo que posibilita la academia.

\section{Conclusiones y recomendaciones}

Se deben valorar significativamente los diferentes contextos (familia, grupo de pares y entorno educativo) que median en la construcción y resignificación de la masculinidad, puesto que en dichos contextos se recrean los conocimientos y las expectativas que derivan en modos particulares de ser hombre.

Las relaciones de intercambio que se configuran en el contexto universitario permiten que los estudiantes asuman una postura crítica respecto a los discursos y prácticas asociadas a la masculinidad hegemónica, de ahí que contemplen la necesidad de trascender algunas prácticas con las que no se sienten satisfechos, aunque se torne complejo el desprendimiento de estas, dada la presión social a la que están expuestos.

Por lo anterior, es importante seguir avanzado en la construcción de relaciones equitativas respecto al género, procurando la no discriminación de las masculinidades que emergen a partir de la crítica y resignificación de la masculinidad hegemónica.

Comprender la resignificación de la masculinidad hegemónica posibilita el reconocimiento de formas múltiples y diversas respecto al ser hombre, de ahí que sea necesario seguir avanzando en las reflexiones sobre las masculinidades a través de este tipo de estudios en contextos educativos. Lo anterior permitirá la construcción de dispositivos pedagógicos que incentiven el respeto por la diferencia desde la igualdad.

Como recomendaciones se espera que desde la universidad se consolide una política institucional de género que facilite la construcción de planes, programas y proyectos que contribuyan al reconocimiento de la diversidad y la equidad de género en el contexto universitario.

Además, se recomienda que los programas académicos generen espacios de discusión, análisis y reflexión de manera permanente, que contribuyan en la generación de prácticas pedagógicas y asignaturas electivas que involucren la perspectiva de género, la equidad y la inclusión social, como aspectos indispensables para la formación, y de esta manera, aportar al cumplimiento de los objetivos misionales de la universidad.

Finalmente, se espera dar continuidad al estudio sobre las masculinidades para poner 
en discusión las miradas y significados sobre el ser hombre, teniendo en cuenta una perspectiva relacional; es decir, se requiere avanzar en la comprensión de cómo las mujeres significan las diferentes formas de masculinidad, además de reconocer la influencia del contexto universitario en las identidades de género.

\section{Referencias}

Aguayo, F., Correa, P. y Cristi, P. (2011). Encuesta IMAGES Chile Resultados de la Encuesta Internacional de Masculinidades y Equidad de Género. Cultura Salud/EME.

Ale, I. (2010). La construcción de las masculinidades entre adolescentes trabajadores de Lima, Perú. Revista Internacional de Ciencias Sociales y Humanidades, SOCIOTAM, XX(1), 161-177.

Barba, Á. y Gómez, R. (2016). Percepciones acerca de la masculinidad en un grupo de hombres y mujeres del Área Metropolitana de Bucaramanga - Santander, Colombia. Reflexión Política, 18(36), 212-223.

Berger, P. y Luckmann, T. (1986). La construcción social de la realidad. Amorrortu.

Bourdieu, P. (1991). El sentido práctico. Taurus.

Bruner, J. (1991). Actos de significado: Más allá de la revolución cognitiva. Alianza Editorial.

Burbano, J., Gafaro, M., Torres, A. y Domínguez, M. (2019). Masculinidades en tránsito: las voces de los motociclistas. Masculinidades y Cambio Social, 8(2), 145-170. http:// doi. org/ 10.17583/MCS.2019.3933

Castro, B. J. (2016). Construcción y transformación de masculinidades de los corteros de caña de azúcar del Valle del Cauca. Revista Colombiana de Sociología, 39(1), 79-102.
Catacolí, C., Rodríguez, J. y Correa, N. (2018). Configuración de las relaciones de género. Fundación Universitaria Católica Lumen Gentium.

Connell, R. y Messerschmidt, J. (2005). Hegemonic Masculinity: Rethinking the Concept. Gender and Society, 19(6), 829-859.

Faur, E. (2004). Masculinidades y desarrollo social. Las relaciones de género desde las perspectivas de los hombres. UNICEF Colombia, Arango Editores.

Flecha, R., Puigvert, L. y Ríos, O. (2013). Las nuevas masculinidades alternativas y la superación de la violencia de género. International Multidisciplinary Journal of Social Sciences, 2(1), 88-113.

Gittings, L. (2016). 'When you visit a man you should prepare yourself': male community care worker approaches to working with men living with HIV in Cape Town, South Africa. Culture, Health \& Sexuality, 18(8), 936-950. https:// doi.org/ 10.1080/ 13691058.2016.115 0513

Gökariksel, B. y Secor, A. (2017). Devout Muslim masculinities: the moral geographies and everyday practices of being men in Turkey. Gender, Place \& Culture, 24(3), 381-402.https:/ / doi.org/ 10.1080/0966369X.2017.1314948

Heilman, B., Barker, G. y Harrison, A. (2017). La caja de la masculinidad: un estudio sobre 10 que significa ser hombre joven en Estados Unidos, el Reino Unido y México. Promundo-US y Unilever.

Hernández, R. (2014). Metodología de la Investigación. McGraw-Hill.

Instituto Vasco de la Mujer [Emakunde] (2008). Los hombres, la igualdad y las nuevas masculinidades. Printek. 
Manzelli, H. (2006). Sobre los significados de ser hombre en varones jóvenes en el área metropolitana de Buenos Aires. Revista Estudios Feministas, 14(1), 219-242. https:// doi. org/ 10.1590/ S0104-026X2006000100012

Medina, J. F., Marín, V. y Dávila, C. D. (2018). Vivencia de la masculinidad en hombres de tres generaciones [trabajo de grado de pregrado]. Pontificia Universidad Javeriana.

Messerschmidt, J. (2010). Engendering Gendered Knowledge: Assessing the Academic Appropriation of Hegemonic Masculinity. Men and Masculinities, 15(1), 56-76. DOI: 10.1177/ 1097184X11428384

Muñoz, H. (2015). Hacerse hombre. La construcción de masculinidades desde las subjetividades: un análisis a través de relatos de relatos de vida de hombres colombianos [Tesis de doctorado]. Universidad Complutense de Madrid, España.

Organización Panamericana de la Salud (OPS) (2018). Comunicaciones breves relacionadas con la sexualidad. Recomendaciones para un enfoque de salud pública. Organización Panamericana de la Salud. https:// iris. paho. org/ handle/ 10665.2/ 49504

Ortega, M., Centeno, R. y Castillo, M. (2005). Masculinidad y factores socioculturales asociados al comportamiento de los hombres: Estudio en cuatro países de Centroamérica. UNFPA y CEPAL.

Peña, J. C. (2008). Construcción de masculinidades igualitarias atractivas. La transmisión de valores a través de actos comunicativos en contextos [Tesis de doctorado]. Universidad de Barcelona, España.
Saavedra, P. B. (2013). Concepción de masculinidades en jóvenes universitarios: Sobre ser hombre y cómo se aprende a serlo. Estudio de caso del Hogar de Estudiantes Universitarios de Curicó [Trabajo de grado de pregrado]. Universidad de Chile, Chile.

Sagaró, N. M. y Moraga, A. (2015). Creencias acerca del sexo, el género y la masculinidad en adolescentes de distintos niveles de enseñanza. MEDISAN, 19(10), 1216-1223.

Sandoval. K. (2014). Del dicho al hecho. Las ideologías de género que sustentan las masculinidades hegemónicas. La Manzana de la Discordia, 9, 57-73.

Sanfélix, J. (2011). Las nuevas masculinidades. Los hombres frente al cambio en las mujeres. Prisma Social, 7, 220-247.

Schutz, A. y Luckmann, T. (2003). Las estructuras del mundo de la vida. Amorrortu.

de Sola, A., Martínez, I. y Meliá, J. L. (2003). El cuestionario de actitudes hacia la igualdad de géneros (CAIG): elaboración y estudio psicométrico. Anuario de Psicología, 34(1), 101-123.

Téllez, A. y Verdú, A. (2011). El significado de la masculinidad para el análisis social. Revista Nuevas Tendencias en Antropología, 2, 80103.

Tovar, C. y Pavajeau, C. (2010). Hombres en situación de desplazamiento: transformaciones de la masculinidad. Revista de Estudios Sociales, 36, 95-102.

Villanueva, A. (2011). «Hombre» es una palabra con muchos significados... y es bien difícil serlo. Construcción de identidades masculinas en estudiantes de Ingeniería. [Tesis de maestría]. Universidad de Chile. 
Viveros, M. (2002). De quebradores y cumplidores. Sobre hombres, masculinidades y relaciones de género en Colombia. Universidad Nacional de Colombia, Facultad de Ciencias Humanas, Centro de Estudios Sociales.

Wilson, N., Parmenter, T., Stancliffe, R. y Shuttleworth, R. (2013). From diminished men to conditionally masculine: sexuality and Australian men and adolescent boys with inteIlectual disability. Culture, Health \& Sexuality, 15(6), 738-751. https:// doi.org/ 10.1080/ 1 3691058.2013.780262 